\title{
THE LEVER OSCILLATOR FOR USE IN HIGH RESISTANCE RESONATOR APPLICATIONS *
}

\author{
Kurt O. Wessendorf \\ Sandia National Laboratories Dept. 2254 \\ Albuquerque New Mexico 87185-5800
}

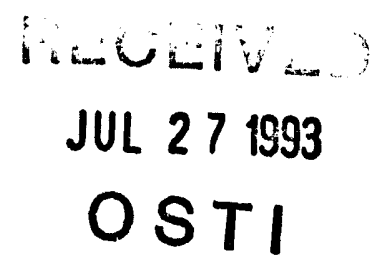

\begin{abstract}
The Lever oscillator has been specifically designed for use with quartz resonator sensors. The use of quartz resonators as sensors is of particular interest and depending on the sensing environment, e.g., liquid, the oscillator design is both critical and difficult due to the wide dynamic range of resonator resistance possible due to damping of the resonator. Standard oscillator designs do not work well as sensor oscillators. An oscillator design will be presented that allows both frequency and loss $\left(R_{m}\right)$ of the resonator to be determined over a wide dynamic range of resonator loss. The Lever oscillator uses negative feedback in a differential amplifier configuration to actively and variably divide (or leverage) the resonator impedance such that the oscillator can maintain the phase and gain of the loop over a wide range of resonator resistance.
\end{abstract}

\section{Introduction}

Standard oscillator designs, like the Pierce or Colpitts type, are good for frequency source oscillator designs which provide low phase noise and high over-all frequency stability. These designs do not necessarily work well for sensor applications because of their inherent phase and gain sensitivity $t o$ resonator loss, $R_{m}$. Operating the resonator at $f_{s}$ is a good initial approach if one is to design an oscillator for use with a high loss resonator. At $f_{s}$ the resonator is approximately a real inpedance and is at or near the minimum impedance of the resonator. Figun: 1 is the model for a bulk wave resonator, with $f_{s}$ defined as the resonance of $L_{m}$ and $C_{m}$. Since a high loss resonator is not likely to be highly inductive at any frequency, it would be indesirable to try to make the sensor operate at an inductive impedance as this will limit the dynamic range of the oscillator. It is also desirable to design this type of oscillator with one side of the resonator at a.c. ground. With one side of the resonator grounded the parasitic capacitances due (o) the fluid can be more easily controlled; this is especially true if only one side of the resonator is used for liquid exposure.

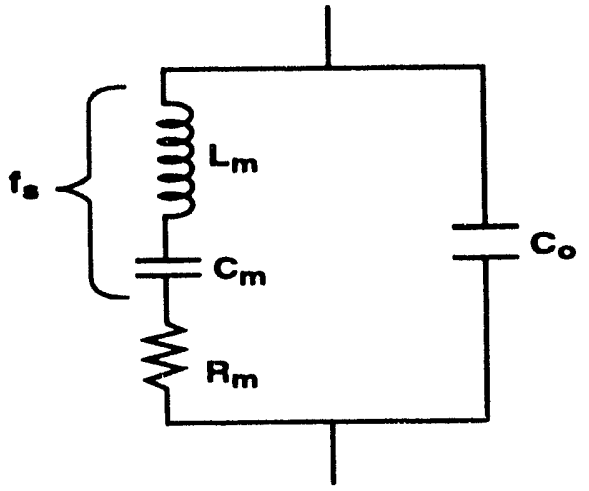

Figure 1 . The quartz resonator model.

\section{Desion Goals}

There are a few oscillator configurations which operate the resonator near $f_{s}$ and have one side of the resonator grounded. None of these configurations meet the design goals of operating at $f_{s}$ of the resonator and oulputting a voltage proportional to the resonator loss $R_{m}$, by way of an automatic level control (ALC) circuit. For in situ resonator sensors the $R_{m}$ of the resonator will have a wide dynamic range. This dynamic range depends upon the resonator and liquid properties but could be tens of ohms in air to several thousand ohms in a viscous liquid. The resonator loss is a function of the viscosity-density product (1). Since quartz resonator frequency shifts due to a given environment are

* This work performed at Sandia National Laboratories by the U.S. Department of Energy under contract DE-AC.0476DP00789

\section{MASTER}


determined by calculating the change in $\mathrm{f}_{\mathrm{s}}$ of the resonator, it is convenient to design the oscillator to operate as close to $f_{s}$ as possible such that the oscillator frequency mirrors the sensor-related frequency changes. The ability to operate at exactly $f_{s}$ of a resonator is in itself a difficult task and the author has found that on an absolute basis it is impossible. In general if the impedance of $C_{o}$ is relatively large compared to $R_{m}$ then $f_{s}$ is close to the frequency at which the resonator impedance phase is zero. The $R_{m}$ output voltuge can also be used to determine the frequency error of the oscillator itself relative to true $f_{s}$ if the shunt capacitance across the resonator is relatively constant throughout the measuring medium.

\section{The Oscillator Desion}

Figure 2 is schematic of an oscillator that evolved into what is called the Lever oscillator. Understanding this circuit will help in understanding how the design goals were ultimately met.

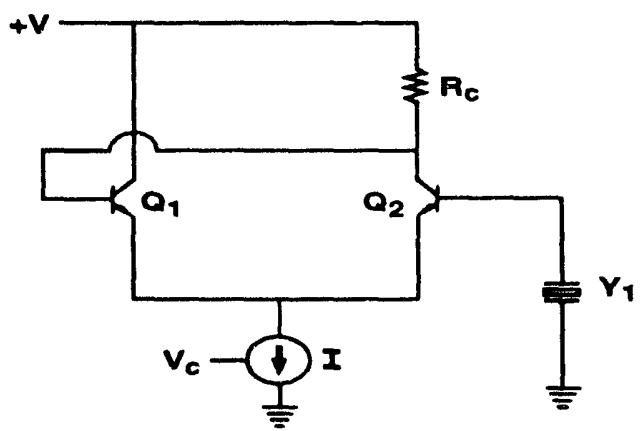

Figure 2. The non-inverting amplifier as an oscillator.

In this oscillator the collector of $\mathrm{Q}_{2}$ is directly connected to base of $Q_{1}$ and the resonator is connected from the base of $Q_{2}$ to ground. The resonator will control (porly) the gain and phase of the potential oscillation. The current source $I$ is controlled by $\mathrm{V}_{\mathrm{c}}$ and will be used in an ALC circuit to servo a given oscillation amplitude and thus will reflect the resonator loss. Transistor $h$ is the reciprocal of transconductance $g_{m}$ and is equal to $(26 \mathrm{mV}) \Omega_{\mathrm{e}}$ where $\mathrm{I}_{\mathrm{e}}$ is the individual transistor bias current. To det rmine the lox)p gain equations one could open the loop at the base of $\mathrm{Q}_{1}$ and determine the loop gain equation for this amplifier, Figure 3 . If the output impedance of the circuit is low relative to the input impedance the gain equation will he fairly representative of the actual oscillator. Equation (1) is the open loop gain equation for the circuit in Figure 3. This circuit, when $V_{\text {out }}$ is connected $t o V_{\text {in }}$, will oscillate when $A_{v}$ is greater than or equal io one.

$$
A_{V} \approx \frac{R_{\mathrm{C}}}{2 h+\frac{Z_{\text {Tes }}}{\beta}}
$$

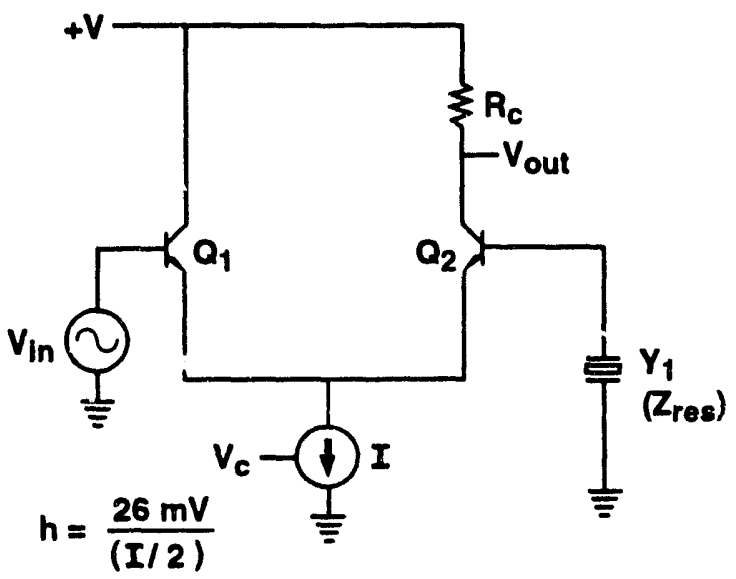

Figure 3. Open lox)p circuit for oscillator calculations.

If $\mathrm{Q}_{1}$ and $\mathrm{Q}_{2}$ are hiased at reasonable current levels ( 0.1 $<$ I $<10 \mathrm{~mA}$ ), the resonator will weakly control the oscillator because the resonator impedance is divided by the transistor $\beta$. Since the loop gain is more dependent on transistor $h$ than the resonator impedance the oscillator sensitivity on resonator resistance is poxor and the lok)p phase of the circuit will not be very sensitive to the resonator impedance. This circuit would work well for the application described if the resonator impedance was effectively divided by a smaller number ( less than $\beta$ ) and was independent of transistor $\beta$. For example it would be desirable to have the resonator impedance divided by a large number when the resonator is a high impedance and divided by a smaller number when the resonator is a low impedance. This variable divider would help to compress the dynamic range of the oscillator transconductance over a wide dynamic range of resonator loss while still allowing enough sensitivity to determine the resonator loss via an automatic level control (ALC) circuit. This function can be obtained by modifying the oscillator of Figure 3 by placing a feedback resistor from the collector of $Q_{2}$ to the base of $Q_{1}$. The Lever oscillator design (open lox)p) can be seen in Figure 4. This schematic shows the feedback resistor, $R_{f}$, that is used to make the oscillator sensitivity to resonator impedance a function of the resonator impedance itself. This function can be made $\beta$ independent and will allow the resonator impedance to be effectively divided (leveraged) as a function of the magnitude of the resonator impedance. 


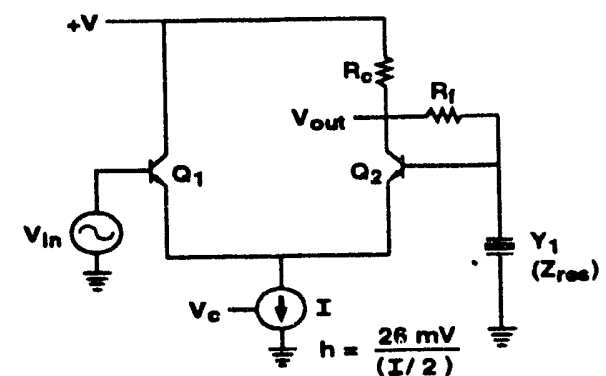

Figure 4. The Lever oscillator open loop circuit.

The Lever oscillator open loon gain, $A_{v}$ if $R_{c}<R_{f}$ :

$$
A_{v} \approx \frac{R_{c}}{2 h+\frac{Z_{\text {Tes }} \|\left[R_{f}+R_{c}\right]}{\beta}+\frac{R_{c} Z_{\text {Tes }}}{R_{c}+R_{f}+Z_{\text {Tes }}}} ;
$$

for oscillation $A_{v}$ must be greater than or equal to one. If all the variables in equation (2) are real, then $Z_{T e s}$ must also be real (have a zero impedance phase). If $Z_{\text {Tes }}$ is real then the frequency of operation is close to $f_{s}$ of the resonator and the resonator impedance can be approximated by $R_{m}$. Equation (2) can be simplified to:

$$
A_{v} \approx \frac{R_{\mathrm{c}}}{2 h+\frac{R_{c} R_{m l}}{R_{c}+R_{\mathrm{f}}+R_{m}}} \geq 1
$$

where $\beta$ is considered large enough to make the center term in the denominator of the loop gain equation (2) very small in comparison to the other two terms. The terin

$$
\frac{R_{\mathrm{c}} R_{\mathrm{m}}}{R_{\mathrm{c}}+\mathrm{R}_{\mathrm{f}}+\mathrm{R}_{\mathrm{m}}}
$$

is the "Lever" term, since it will determine how the resonator impedance is effectively divided (leveraged) by the feedback circuit elements, $R_{c}$ and $R_{f} \cdot R_{m}$ is effectively divided by the function

$$
\frac{R_{\mathrm{c}}+R_{\mathrm{f}}+R_{\mathrm{ln}}}{R_{\mathrm{c}}} \equiv g\left(R_{\mathrm{m}}\right)
$$

which is small when the resonator impedance is small and large when the resonator impedance is large. The Lever term (4) is transistor $\beta$-independent and can be made to dominate the given gain equation (2) over a wide range of $\beta$. This variable leverage function allows the oscillator to operate over a wider dynamic range of resonator resistance while maintaining sensitivity at lower resonator resistances than if the divider effect was constant or dependent on $\beta$. Figure 5 shows a graph of the equation (5) vs $R_{m}$. This graph represents the variable divider effect the circuit has on $R_{m}$ with $R_{c}$ equal to $150 \mathrm{ohms}$ and $R_{f}$ equal to $1000 \mathrm{ohms}$. Due to the fact that the circuit is operating close to $f_{S}$ of the resonator, $Z_{\text {Tes }}$ is approximately $R_{m}$ if $C_{o}$ is relatively small.

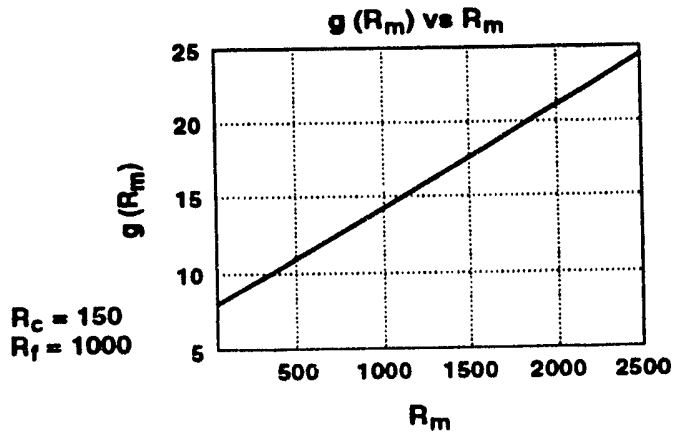

Figure 5. Graph of variable "Lever effect", $g\left(R_{m}\right)$ vs $R_{m}$.

For the values of $R_{c}$ and $R_{f}$ chosen, the resonator impedance $R_{m}$ is effectively divided by 7 for small values of $R_{m}$ and by approximately 24 for large values of $R_{m}$. By selecting the values of $R_{f}$ and $R_{c}$ such that $R_{f} / R_{c} \ll \beta$, the oscillator will he relatively independent of $\beta$. The choice of $R_{f}$ and $R_{c}$ in combination with the current source function $\left(V_{c}\right)$ allow the designer to set the oscillator sensitivity to a desired range of $\mathbf{R}_{\mathbf{m}}$.

\section{Lever Oscillator Practical Realization}

The oscillator theory presented to this point has virtually ignored transistor capacitances and the practical matters of the design. Figure 6 is a schematic of a practical form of the Lever oscillator.

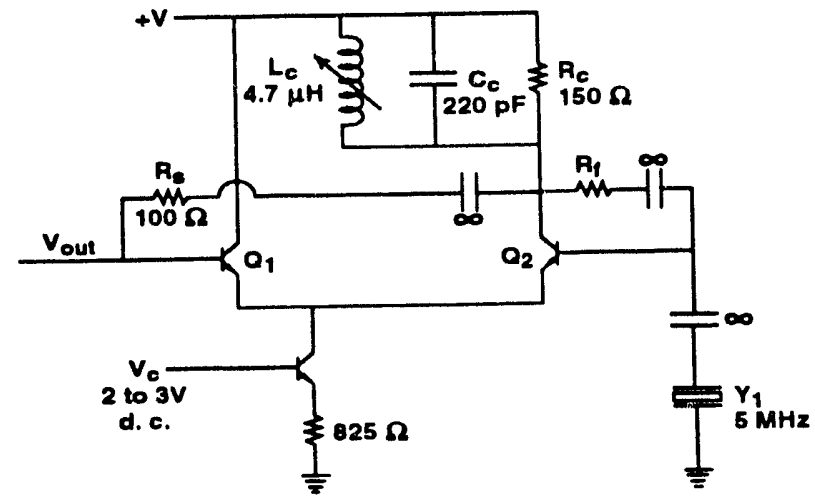

Figure 6. Lever oscillator schematic. 
This schematic will be used to describe and derive the actual design performance of the oscillator function. In the theory stated above it was stated that if all the circuit components shown (Figure 4) were real then at the oscillation frequency the resonator would also be real. But in actuality, at frequencies greater than d.c., circuit capacitance will influence circuit performance. Capacitance from collector to ground of $Q_{2}$ combined with base to ground capacitance of $Q_{1}$ must be addressed. This capacitance, lumped together because of the circuit configuration, significantly degrades circuit performance: (1) this capacitance pulls the oscillator low in frequency (even for a small amount of capacitance) and (2) if the resonator becomes highly lossy, the circuit has a tendency to jump to a parasitic (capacitor controlled) frequency. These two problems were minimized by placing a parallel L-C $\left(L_{c}\right.$ and $C_{c}$ ) tank circuit across $R_{c}$. This tank is designed to be low $Q$ (1 to 3) and is used to make the collector to ground impedance at $Q_{2}$ to be real (approximately $R_{c}$ ) at the desired frequency and to help constrain the oscillation frequency $(0)$ the resonator and not to a parasitic frequency. This tank is adjusted such that with a resistor (low value, 100 ohms) in place of the resonator the tank circuit is adjusted to make the oscillator frequency approximately equal to the desired resonator frequency. With $R_{\mathbf{c}}$ real at the resonator frequency we have satisfied one of the assumptions given in the theory above.

The other assumption in the theory is that $R_{f}$ is also real. Since $\mathrm{R}_{\mathrm{f}}$ is a major component in the design, it is interesting to see the effect of capacitance across $R_{f}$ due $t$ o the $C_{b c}$ of the transistor. By using equation (3), substituting $Z_{\text {res }}$ for $R_{m}$ and $Z_{f} f(r) R_{f}$ one can solve for $Z_{r e s}$ and determine the phase and magnitude of $Z_{\text {res }}$ as a function of $Z_{f}, R_{c}$ and $h$.

$$
\mathrm{Z}_{\mathrm{res}} \approx \frac{\left[\mathrm{R}_{\mathrm{c}}-2 \mathrm{~h}\right]\left[\mathrm{R}_{\mathrm{c}}+\mathrm{Z}_{\mathrm{f}}\right]}{2 \mathrm{~h}}
$$

and if $\mathrm{R}_{\mathrm{c}}<\mathrm{Z}_{\mathrm{f}}$

$$
\mathrm{Z}_{\mathrm{res}} \approx \frac{\left[\mathrm{R}_{\mathrm{c}}-2 \mathrm{~h}\right]\left[\mathrm{Z}_{\mathrm{f}}\right]}{2 \mathrm{~h}}
$$

where $R_{c}$ and $h$ are real. If these assumptions are $g(x) d$ then it can be seen from equation (7) that the resonator impedance phase must be equal to the phase of $\mathrm{Z}_{\mathrm{t}}$. If operating close $(0$ zero impedance phase of the resonator is desired it is important to s $p$ lect $R_{f}$ such that $R_{f}$ is much less than the impedance of $C_{b c}$ for the chosen transistor. If one was to design this oscillator with a transistor having a $C_{b c}$ of approximately 3 $\mathrm{pF}$ and $R_{f}$ of 1000 ohms then the impedance, $Z_{f}$ for $R_{f}$ in parallel with $3 \mathrm{pFat} 6 \mathrm{MHz}$, will equal 994 (shms at $-6.45^{\circ}$. For this case the resonator impedance phase at the oscillation frequency will also be approximately $6^{\circ}$. Although the equations derived throughout this paper are for small-signal low-excess loop-gain (one) conditions, the oscillator essentially behaves as equation (6) predicts; this is true for two reasons: (1) the oscillator will be used in an ALC circuit which is used to control the excess gain to a relatively low value so that $R_{m}$ can be determined and (2) the differential amplifier limiting characteristics provide a symmetrical limiting action that makes the circuit behave more linearly than a simple single-transistor oscillator circuit. Figure 7 is a calculation of resonator impedance and phase versus transisior $h$ using equation (6).

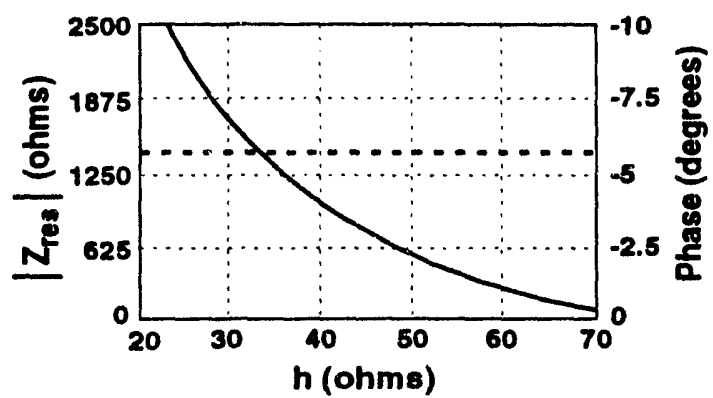

Figure 7. Calculated resonator magnitude and phase vs $h$ calculations for the Lever oscillator.

These graphs demonstrate the wide dynanic range of resonator impedance possible with the oscillator controlling the crystal inpedance phase to approximately the phase of the feedhack impedance $\mathrm{Z}_{\mathrm{f}}$.

\section{Lever Oscillator Performance Data}

To indirectly measure resonator resistance an ALC. Ioxp was designed to control the oscillator gain (h) via the voltage controlled current source, $Q_{3}$, (Figure 8 ) of the oscillator.

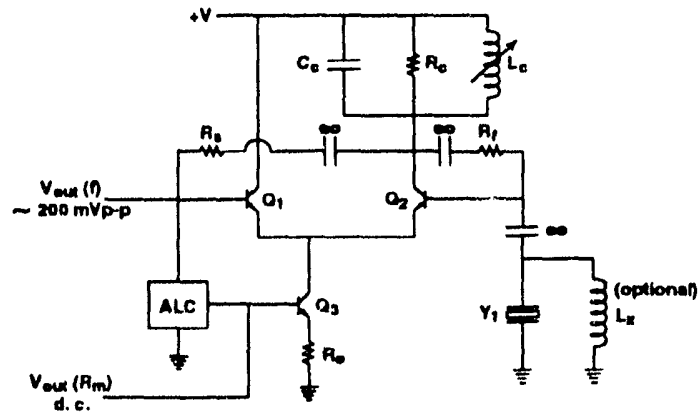

Figure 8. The Lever oscillator with ALC circuit. 
The ALC was designed to control the oscillation amplitude at the base of $Q_{1}$ to approximately $200 \mathrm{mV}$ peak-t()-peak. Figure 8 also shows an inductor $L_{x}$ which can be used to tune out static capacitance of the fixture and resonator. $\mathrm{L}_{x}$ can dramatically increase the upper resistance limit hefore the oscillator breaks into a parasitic oscillation. To evaluate the Lever oscillator for one set of circuit parameters, a $6 \mathrm{MHz}$ test resonator was made using a standard low resistance device in series with added resistance. Figure 9 shows the test resonator used to evaluate the Lever oscillator.

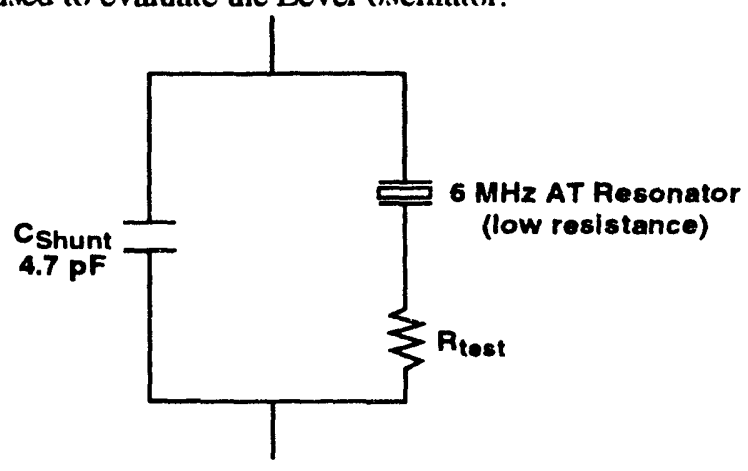

Figure 9. Test resonator with variable resistance.

$C_{\text {shunt }}$ was included in the test resonator to more accurately simulate the $\mathrm{C}_{0}$ of a standard resonator. This resonator was used to determine the actual resonator inpedance phase at oscillation for a wide range of resistance, $R_{\text {test }} \cdot R_{\text {test }}$ was varied from 56 to $1700 \mathrm{ohms}$ and the resonator impedance was measured on an impedance analyzer at the frequency of operation of the Lever oscillator for each tested resistance. Also the d.c. voltage of the ALC. was recorded for all values of $R_{\text {test }}$. Data relating resonator impedance, phase, and d.c. output voltage as a function of $R_{\text {test }}$ are presented in Figures 10 and 11 respectively.

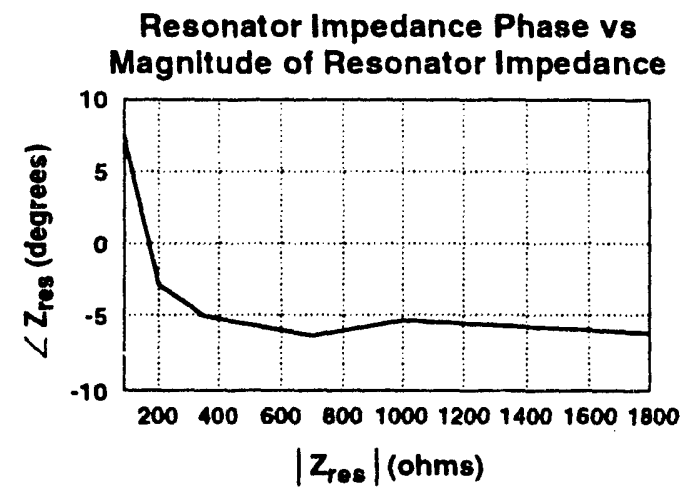

Figure 10. Measured resonator impedance phase vs magnitude.

\section{Oscillator Vout (d.c.) vs Resonator Resistance}

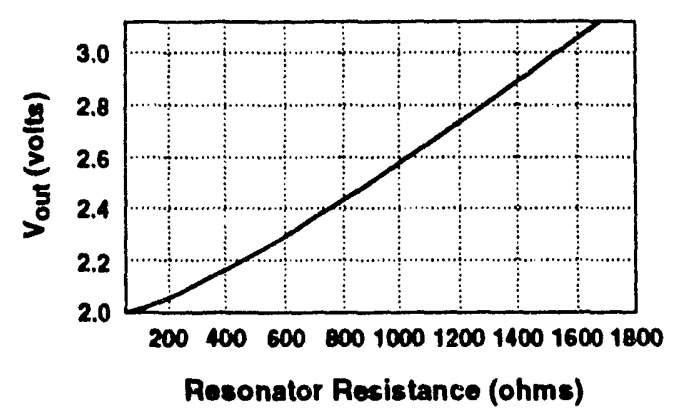

Figure 11. ALC voltage out vs resonator resistance.

These data demonstrate that the oscillator does indeed servo the resonator inpedance to approximately the phase of $\mathrm{Z}_{\mathrm{f}}$, in this case approximately $6^{\circ}$, when the resonator is above 200 ohms. At resonator impedances below $200 \mathrm{ohms}$ the resonator impedance phase increases. This phase shift at low resonator impedances is explained by the fact that the oscillator is less controlled by the resonator when the resonator impedance is low than when large and is therefore more sensitive to circuit parameters not used in the approximations presented here. To illustrate this, a more exact model of the oscillator was used which assumed the transistor $h$ to be very slightly capacitive. When this capacitance is included in the calculations the resonator impedance phase increases with decreasing resonator impedance just as seen in the data, Figure 10. Figure 12 shows the calculated magnitude of $Z_{\text {res }}$ versus the phase of $Z_{\text {Tes }}$ wit' 1 transistor $h$ slightly capacitive.

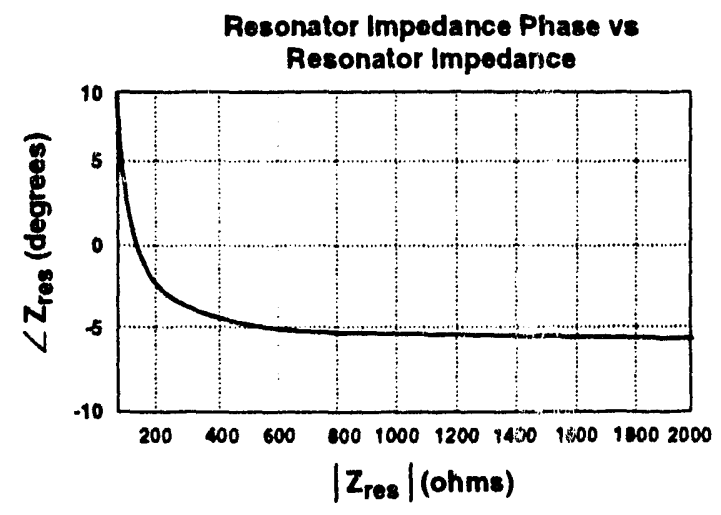

Figure 12. Calculation of resonator impedinnce phase vs magnitude for h slightly capacitive. 
To demonstrate the $\beta$ insensitivity of the Lever oscillator, a negative resistance model was used to calculate the input impedance $\left(Z_{\text {in }}\right)$; the calculations are shown in Figure 13 as a function of $h$ for transistor $\beta$ 's of 150 and 50 .

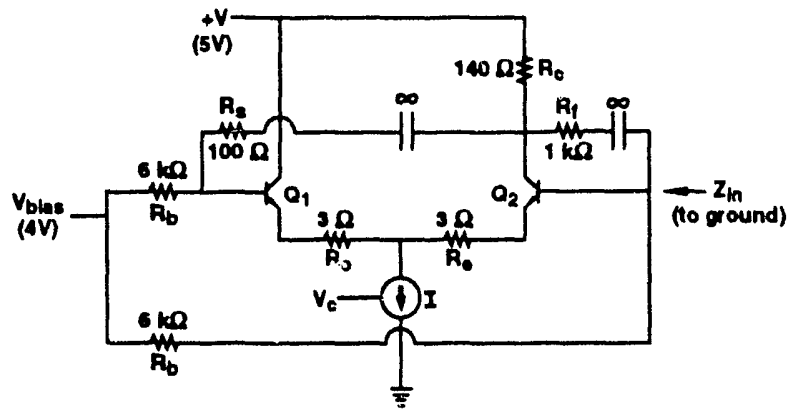

Figure 13. Lever oscillator circuit used in negative model calculations.

Figure 14 is a graph of the negative resistance function of the lever oscillator for $\beta$ equal to 50 and 150 . The oscillator $\beta$ sensitivity is greatest at high resonator resistances where the negative feedback due to $R_{f}$ and $R_{c}$ is least effective. With the feedback used in this example the oscillator is relatively insensitive to transistor $\beta$ for $h$ between 20 and 6.5 ohms.

\section{Oscillator $Z_{\text {in }}$ vs Transistor $h$}

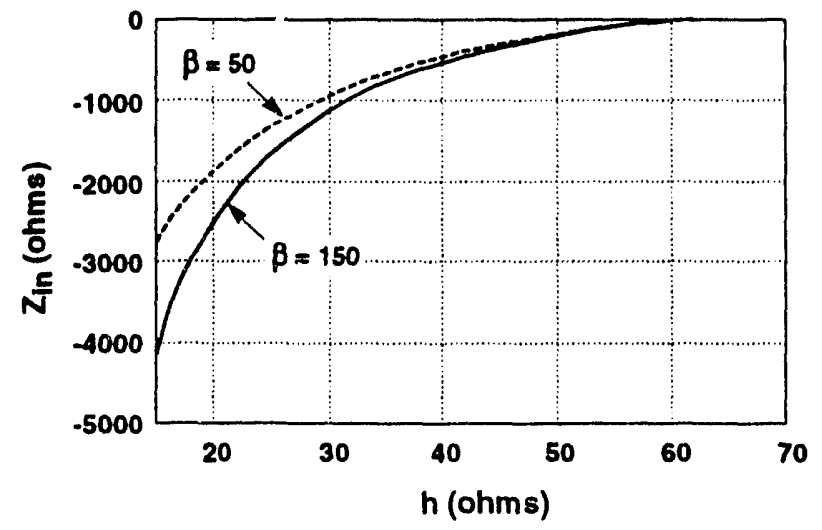

Figure 14. Calculation of $\beta$ sensitivity vs $h$.

\section{Qscillator Erequency Error}

Now that it has been established that the Lever oscillator function can be made to act like an impedance phase servo it would be interesting to determine how this phase frequency relates to the true $\mathrm{f}_{\mathrm{s}}$ of the resonator. If $\mathrm{C}_{\mathrm{o}}$ is zero then $\mathrm{f}_{\mathrm{s}}$ is equal to the zero impedance phase frequency. But since $C_{0}$ is never zero then the oscillation frequency of the Lever oscillator will he offset from true $f_{s}$ of the resonator by some amount depending on $R_{m}, C_{o}$, and the phase of the resonator impedance which the oscillator is servoing. Figure 15 is a graph showing the calculated difference between the frequency of zero impedance phase of the resonator and $f_{s}$ of the resonator. This is done for two static capacitances of the resonator, $1 \mathrm{pF}$ and $3 \mathrm{pF}$. The graph shows that, for $\mathrm{C}_{0}$ equal to $1 \mathrm{pF}$, the frequency error with $\mathrm{C}_{\mathrm{m}}$ equal to $25 \mathrm{fF}$ and $\mathrm{L}_{\mathrm{m}}$ equal to $31 \mathrm{mH}$ is relatively small hut increases with resonator resistance $R_{\mathbf{m}}$.

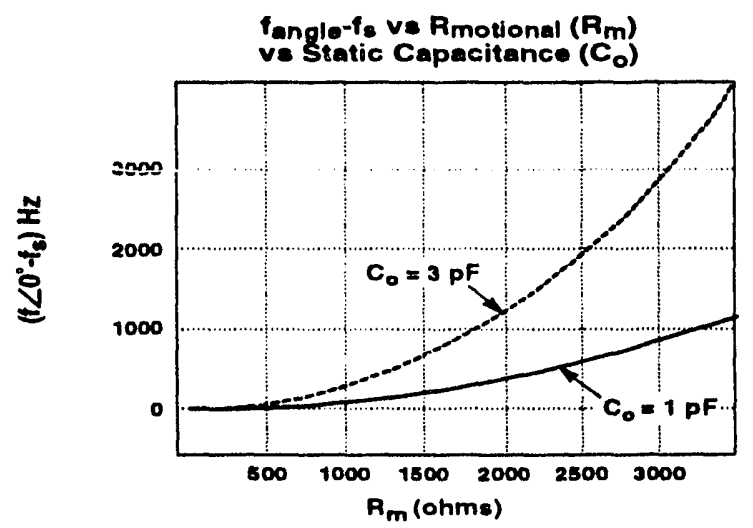

Figure 15. Frequency at ()$^{\circ}$ phase $-f_{\mathrm{s}}$ vs $R_{\mathrm{m}}$.

With increasing $C_{0}$ the frequency error increases Jranatically with $R_{m}$. Figure 16 is a graph of the same function but in this case a difference frequency for $-6^{\circ}$ of impedance phase and $\mathrm{f}_{\mathrm{s}}$ is calculated and the resulting curve shows a negative error for small $R_{m}$, sweeping positive as the resistance increases.

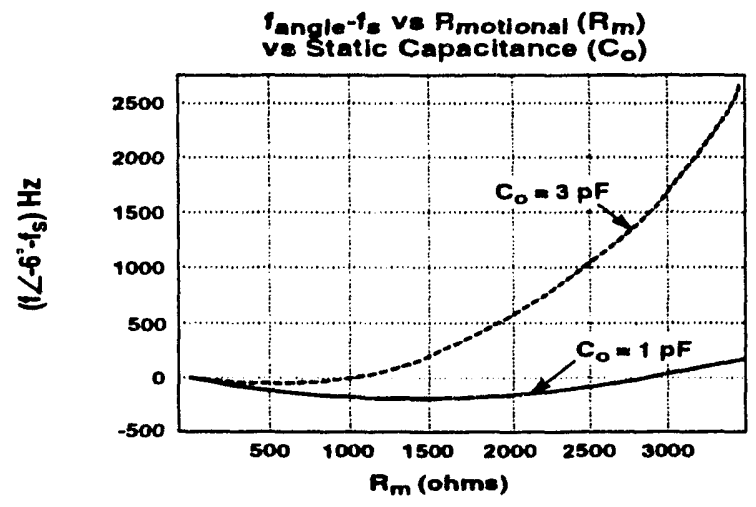

Figure 16 . Frequency at $-6^{\circ}$ phase $-f_{s}$ vs $R_{m}$. 
This is to be expected since at $f_{\mathrm{s}}$ the resonator is essentially a capacitance $\left(C_{0}\right)$ in parallel with resistance $\left(R_{m}\right)$. Therefore, the resonator impedance phase at $f_{s}$ is a negative quantity varying with $R_{m}$ and/or $C_{0}$. To make a "true" $f_{s}$ oscillator one would have to vary the phase that the oscillator servos as a function of $R_{m}$ given a fixed $C_{0}$. Due to the fact that the Lever oscillator has a $R_{m}$-sensitive output voltage, one can use this output to correct for the frequency error calculated in Figures 15 and 16 if such accuracy is required. This correction could be implemented using digital analog techniques.

\section{Conclusions}

A series resonant oscillator design has been presented that operates over a wide dynamic range of resonator resistance. This resistance range is compatible with in situ resonator sensors used in liquid environments. The Lever oscillator is useful for these applications because of its ability to measure the $f_{s}$ and $R_{m}$ of the resonator. Also, the oscillator's variable leveraging effect has been shown to be useful for increasing the dynamic range of resonator resistance while maintaining sensitivity at lower resistances. The oscillator's ability to servo a resonator-impedance phase has been demonstrated and this phase can be made close to zero degrees by selection of transistor type and/or circuit resistance values. The relationship between the resonator frequency at a given phase with respect to true $f_{s}$ was discussed along with correction methoxls. Currently this design is being implemented in a jet-fuel contamination sensor system and in a viscosity-density sensor using bulk wave resonators (2). Variations on this design have worked with resonators with $R_{m}$ in the 3500 ohm range, at approximately $6 \mathrm{MHz}$, and direct coupled, integratable, versions of this oscillator design are also being develored for applications where small size is important.

\section{Acknowlegements}

The author would like to thank Allyn R. Anderson for his assistance in the development of the Lever ocillator. His fabrication, testing and evaluation of the design helped to make the Lever oscillator a rohust, successful design. Thanks also to Steve J. Martin and Elmer A. Klavetter for giving the author the design choxienge that precipitated the development of the Lever oscillator and for their technical support and encouragement.

\section{Befierences}

(1) S. J. Martin, K. O. Wessendorf, C. T. Gebert, G. C. Frye, C. W. Cernosek, L. Casaus and M. A. Mitchell, "Measuring Lequid Properties With Smoxth-And Textured-Surface Resonators," Proc. 47th Annual Frequency Control Symposium, June 1993.

(2) T. J.O'Hern, W.M. Trott, S. J. Martin and E. A. Klavetter, "Advanced Diagnostics for in situ Measuremeit of Particle formation and Deposition in Thermally Stressed Jet Fuels," AIAA 930363, Aerospace Science Meeting, Reno, Nevada, 1993.

\section{DISCLAIMER}

This report was prepared as an account of work sponsored by an agency of the United States Government. Neither the United States Government nor any agency thereof, nor any of their employees, makes any warranty, express or implied, or assumes any legal liability or responsibility for the accuracy, completeness, or usefulness of any information, apparatus, product, or process disclosed, or represents that its use would not irifringe privately owned rights. Reference herein to any specific commercial product, process, or service by trade name, trademark, manufacturer, or otherwise does not necessarily constitute or imply its endorsement, recommendation, or favoring by the United States Government or any agency thereof. The views and opinions of authors expressed herein do not necessarily state or reflect those of the United States Government or any agency thereof. 

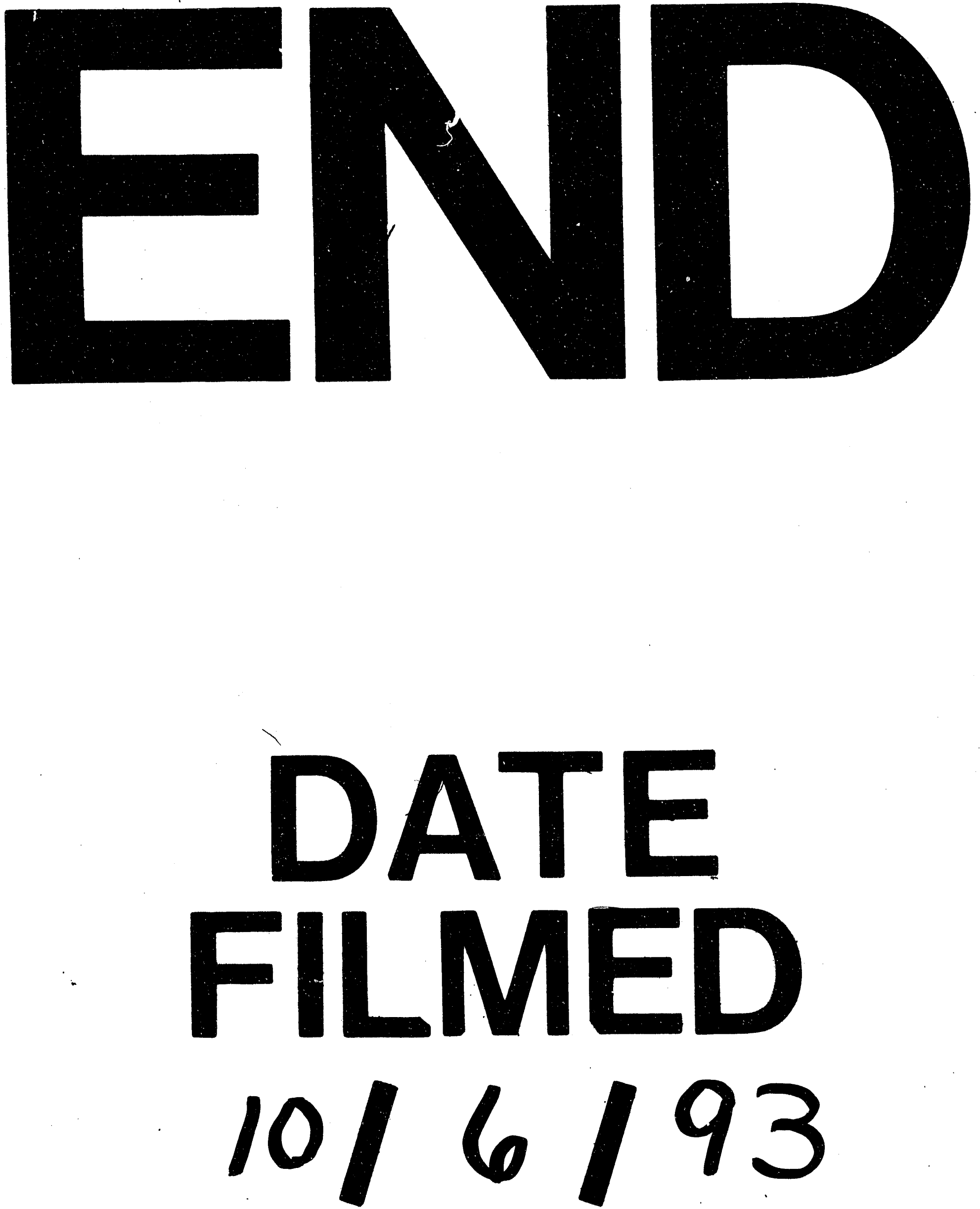
